\title{
Wetting, interfacial interactions and sticking in glass/steel systems
}

\author{
Jérôme Pech ${ }^{\mathrm{a}}$, Muriel Braccini ${ }^{\mathrm{a}}$, Andreas Mortensen ${ }^{\mathrm{b}}$, Nicolas Eustathopoulos ${ }^{\mathrm{a}, *}$

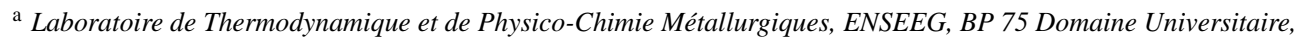 \\ 38402 Saint-Martin d'Hères Cédex, France \\ ${ }^{\mathrm{b}}$ Laboratoire de Métallurgie Mécanique, Ecole Polytechnique Fédérale de Lausanne, 1015 Lausanne, Switzerland
}

Received 2 December 2003; received in revised form 21 May 2004

\begin{abstract}
Wetting and sticking of soda-lime glass on two types of stainless steel as well as on platinum and vitreous carbon substrates are studied in a neutral gas atmosphere between 860 and $1200^{\circ} \mathrm{C}$. Wetting is measured by the "transferred drop" version of the sessile drop technique, enabling fully isothermal spreading kinetics to be monitored. Sticking is investigated by measuring the temperature of glass drop detachment from the substrate during cooling below the vitreous transition temperature. Characterization of substrate and glass surfaces after separation is carried out using surface profilometry, atomic-force microscopy (AFM) and scanning electron microscopy (SEM) with energy-dispersive X-ray (EDX) spectroscopy. The character of molten glass wetting on metal (reactive or non-reactive) and the type of interactions ensuring ultimate wetting and adhesion (physical or chemical) are identified and discussed. The factors controlling glass spreading kinetics and those governing glass/steel sticking are also evidenced.
\end{abstract}

(C) 2004 Elsevier B.V. All rights reserved.

Keywords: Wetting; Bonding; Sticking; Glass

\section{Introduction}

A major problem in glass container production is sticking of the glass on metallic molds. As a consequence, in glass molding by pressing or centrifugation [1], the selection of process parameters such as mold temperature or cooling rate is strongly influenced by the requirement of non-sticking. In the open literature there is no satisfactory theory explaining the mechanism of glass sticking in such processes. Although several studies of mold material wetting by molten glass have been published, (see for instance [2,3]), to the best of our knowledge no theory exists relating sticking with wetting and glass-mold interfacial interaction. It is not possible to formulate such a theory at present because a divergence of experimental results exists concerning the effect on sticking of material parameters such as the mold surface chemistry and roughness [4-7]. One main reason explaining this divergence is the lack of standard experimental techniques to quantify sticking.

\footnotetext{
* Corresponding author. Tel.: +33-476-82-65-04; fax: +33-476-82-67-67.

E-mail address: nikos@1tpcm.inpg.fr (N. Eustathopoulos).
}

This study is a contribution to the subject based on sessile drop wetting and sticking experiments. The results are supplemented by chemical and morphological characterization of mold and glass surfaces in contact. The glass of this investigation is soda-lime glass, which is the most widely used in production. The substrates simulating mold materials are two common stainless steels. Some additional experiments are also performed with platinum and vitreous carbon substrates. Platinum is used as a noble metal reference, while vitreous carbon enables the simulation of mold lubrication, which is often practiced in glass container production. Experiments are carried out in a pure helium atmosphere.

The wetting experiments are performed by the "transferred drop" version of the sessile drop technique, enabling fully isothermal spreading kinetics to be monitored. Sticking is investigated by measuring, during cooling below the vitreous transition temperature $\left(T_{\mathrm{g}}\right)$, the temperature $\left(T_{\mathrm{d}}\right)$ at which the "solidified" glass drop spontaneously detaches from the substrate. The chemistry of substrate and glass surfaces after separation is determined using scanning electron microscopy (SEM) with energy-dispersive X-ray (EDX) spectroscopy. The topography of these surfaces is determined by two complementary techniques, namely surface profilometry and atomic-force microscopy (AFM), so that 
features at different scales from $1 \mathrm{~nm}$ to several $\mu \mathrm{m}$ can be detected. The study parameters are the temperature between 860 and $1200^{\circ} \mathrm{C}$ as well as the surface chemistry of the steel, oxidized and unoxidized. The experiments were carried out with smooth substrate surfaces (average roughness of a few $\mathrm{nm})$. For comparison purposes, experiments were also performed on rough substrate surfaces (of average roughness around $1 \mu \mathrm{m}$ ).

\section{Experimental procedure}

The glass used in this investigation is common soda-lime glass. Its composition is $\mathrm{Na}_{2} \mathrm{O} 13.4, \mathrm{CaO} 10.9, \mathrm{MgO} 1.4$, $\mathrm{Al}_{2} \mathrm{O}_{3} 1.6$ and $\mathrm{SiO}_{2}$ balance in wt.\%. For the wetting and sticking experiments the glass mass was set at around 100 $\pm 3 \mathrm{mg}$ to minimize gravity effects on drop shape and to favor refinement of the glass, as described below.

Among the different mold materials used in glass container production, two widespread stainless steels were selected, commercially designated as RNOS and X25V. The average value of their Young's modulus $(E)$ and thermal expansion coefficient $(\alpha)$ in the range from 20 to $500^{\circ} \mathrm{C}$ are given in Table 1 together with their chemical composition. The steel substrates were $20 \mathrm{~mm}$ in diameter and $3 \mathrm{~mm}$ in thickness. In addition to stainless steel, pure platinum $(\mathrm{Pt})$ and vitreous carbon substrates $(\mathrm{Cv})$ were used. The $99.95 \%$ pure platinum sample was a $15 \mathrm{~mm} \times 15 \mathrm{~mm}$ square with a thickness of $1 \mathrm{~mm}$. The vitreous carbon sample was a $20 \mathrm{~mm}$ $\times 20 \mathrm{~mm}$ square with a thickness of $3 \mathrm{~mm}$, with no open porosity and an ash content less than $50 \mathrm{ppm}$. All steel, Pt and vitreous carbon substrates were polished to an average final roughness of $2 \mathrm{~nm}$. Experiments were also performed using micro-blasted $\mathrm{X} 25 \mathrm{~V}$ surfaces having an average roughness of $1.3 \mu \mathrm{m}$.

In the classical sessile drop technique, a piece of glass is placed on the substrate surface, then heated to the wetting temperature $\left(T_{\mathrm{w}}\right)$. A preliminary experiment was performed using this technique for $T_{\mathrm{w}}=960^{\circ} \mathrm{C}$. During heating the glass started to change shape above about $600^{\circ} \mathrm{C}$. The wetting process was nearly finished once $T_{\mathrm{w}}$ had been reached. Clearly, this technique is not suited to studying the isothermal wetting kinetics of glass. Therefore experiments were carried out using the "transferred drop" variant of the sessile drop technique. In this method the glass is melted on an inert auxiliary substrate, heated to the wetting temperature and then transferred by capillary contact to the substrate to be wetted. Another advantage of this technique, as will be seen below, is that the molten glass on the auxiliary substrate can be refined before transfer.

The experiments were performed in a metallic furnace. The atmosphere was of helium purified through a $\mathrm{Zr}-\mathrm{Al}$ alloy of high specific area to maintain the oxygen partial pressure below $10^{-9} \mathrm{~Pa}$. The furnace consisted essentially of a molybdenum resistance fitted with windows enabling direct illumination of the sessile drop on the substrate. The spreading process was filmed by a video camera connected to a computer on which the images were stored. After the experiment, the contact angle $\theta$ and the drop base radius $R$ were measured directly from the recorded images. The angle measurement was based on the tangent method using suitable software. The accuracy was $\pm 3^{\circ}$ for $\theta$ and $2 \%$ for $R$.

The glass sample was taken from a large piece of glass, taking care to avoid large defects (cracks or gas inclusions) inside the sample. The sample was ultrasonically cleaned with acetone, then introduced inside the furnace onto a flat vitreous carbon substrate. The latter was chosen as the auxiliary substrate because it is not wetted by glass and reacts negligibly with it. The study substrate was placed about 7-8 $\mathrm{mm}$ above the upper glass surface. A high dynamic vacuum was then created to obtain a pressure of around $10^{-5} \mathrm{~Pa}$ and the system was heated gradually. This step aimed to evacuate all gas residues lying between the glass sample and the carbon support. Afterwards a static pressure of purified helium was created and the system was heated again up to $1200^{\circ} \mathrm{C}$. The temperature was kept at this value as long as necessary (typically about $2 \mathrm{~h}$ ) to refine the glass completely by removing any bubbles that had appeared during heating. Subsequently the temperature was lowered to the wetting temperature $\left(T_{\mathrm{w}}\right)$. When $T_{\mathrm{w}}$ was reached, the glass drop on its carbon support was raised so as to initiate contact between its upper surface and the study substrate, gradually forming a pendular bridge between the substrates, Fig. 1 . As spreading of the drop over the substrate surface progressed under the action of capillary forces, the glass bridge

Table 1

Chemical composition and mechanical characteristics of substrate materials

\begin{tabular}{|c|c|c|c|}
\hline Material & Chemical composition (wt.\%) & $\alpha\left(\mu \mathrm{m} \mathrm{m}^{-1} \mathrm{~K}^{-1}\right)$ & $E(\mathrm{GPa})$ \\
\hline RNOS steel ${ }^{\mathrm{a}}$ & Cr 16.26 , Ni 1.88, Mn 0.43 , Si 0.27 , C 0.20 , Fe balance & 20 (austenitic steel) 11 (martensitic steel) & 200 \\
\hline $\mathrm{X} 25 \mathrm{~V}$ steel $^{\mathrm{b}}$ & Cr 25.0, Ni 20.0, Si 2.0, C 0.1, Fe balance & 17 & 180 \\
\hline Platinum [8] & Pt $(99.9 \%)$ & 9 & 250 \\
\hline Vitreous carbon ${ }^{\mathrm{c}}$ & $\mathrm{C}$ (ash content $<50 \mathrm{ppm})$ & 3 & $40^{\mathrm{d}}$ \\
\hline
\end{tabular}

\footnotetext{
a Saarsthal GmbH-Germany.

b Aubert\&Duval SA-France.

c Carbone Lorraine SA-France.

d $E$ of vitreous carbon was determined by normalized four-point bend testing.
} 


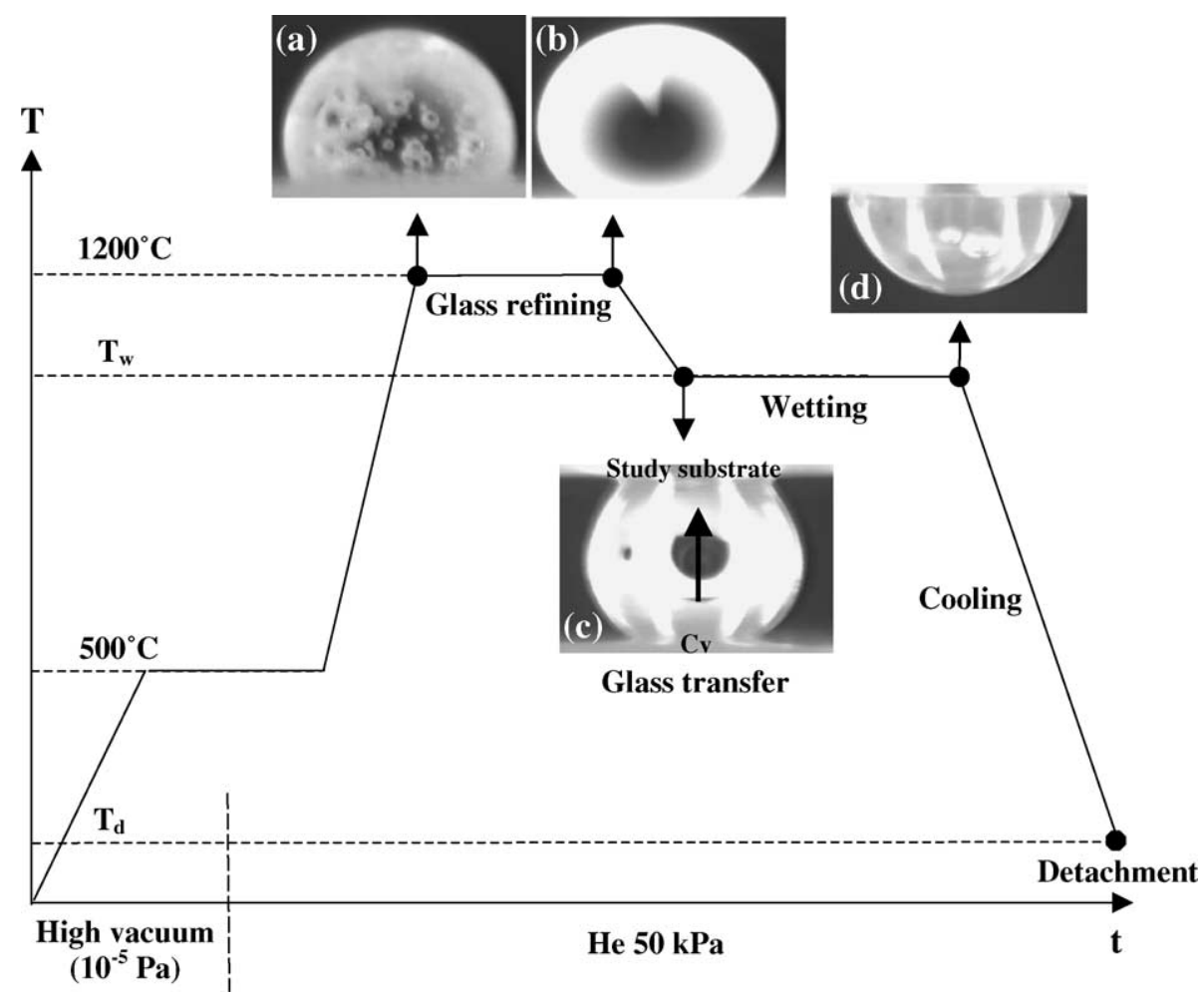

Fig. 1. The different steps of the "transferred drop" variant of the sessile drop technique: (a) non-refined glass; (b) refined glass; (c) glass pendular bridge; (d) hanging glass drop.

abruptly became unstable. At that moment, most of the pendular drop detached from the carbon support to become a sessile glass drop hanging under the study substrate. Once the drop shape had stabilized, corresponding to the final contact angle, the system was cooled down naturally, Fig. 1. At a system-dependent temperature called the "detachment temperature" $\left(T_{\mathrm{d}}\right)$, found to be lower than the vitreous transition temperature $\left(T_{\mathrm{g}} \approx 560^{\circ} \mathrm{C}\right)$ by several hundred degrees, the "solidified" glass drops were observed to detach suddenly from the substrate. As detailed below, the temperature difference $\left(T_{\mathrm{g}}-T_{\mathrm{d}}\right)$ is used as an approximate measure to quantify the sticking of glass on the substrate. The cooling rate around $T_{\mathrm{d}}$ was kept constant in all experiments equal to $-10 \mathrm{~K} \mathrm{~min}^{-1}$. The total glass loss throughout each experiment (glass refinement and spreading process) was less than $0.1 \mathrm{mg}$.

Most experiments were performed under conditions allowing complete deoxidization of steels; however, in glass container production the gas environment is air and steel molds are therefore oxidized. For this reason, it appeared interesting to study the influence of substrate oxidation on $T_{\mathrm{d}}$. A first attempt was made by oxidizing a RNOS substrate for $3.5 \mathrm{~h}$ at $450{ }^{\circ} \mathrm{C}$ in air, which led to the formation of an oxide film about $100 \mathrm{~nm}$ thick. Unfortunately this oxide film evaporated completely after heating in purified helium at $1034{ }^{\circ} \mathrm{C}$ for $30 \mathrm{~min}$. A second attempt was then made by oxidizing the same substrate for $8 \mathrm{~h}$ at $860^{\circ} \mathrm{C}$ to generate an oxide film about $1 \mu \mathrm{m}$ thick. As will be seen later, this oxide film was thick enough to allow a real wetting experiment to be performed on oxidized steel.

Several techniques were employed to determine the roughness and chemistry of the substrate and glass surfaces in contact. A Talysurf surface profilometer was used to measure roughness over areas of several $\mathrm{mm}^{2}$ and an atomic force microscope for characterizing surface evenness defects with areas of several $\mu \mathrm{m}^{2}$. In parallel, a scanning electron microscope with an energy-dispersive X-ray spectrometer was operated to obtain data on local chemistry.

\section{Results}

After preliminary experiments performed at $T_{\mathrm{w}} \geq 960^{\circ} \mathrm{C}$, the steel surface appears to be unoxidized. This is in contrast with experimental observations obtained for $T_{\mathrm{w}} \leq 860^{\circ} \mathrm{C}$, where oxidation of the free metallic surface is evidenced by strong coloration. It is worth pointing out that this oxidation requires direct contact between the glass and the metal. Indeed, an experiment carried out at $T_{\mathrm{w}}=860^{\circ} \mathrm{C}$, by placing the glass drop at about $1 \mathrm{~mm}$ from the steel substrate without direct contact, did not lead to any oxidation of the metallic surface. To avoid the complications due to such steel oxidation by glass at $T_{\mathrm{w}} \leq 860^{\circ} \mathrm{C}$, most wetting and sticking experiments were performed at $T_{\mathrm{w}} \geq$ $960^{\circ} \mathrm{C}$. 


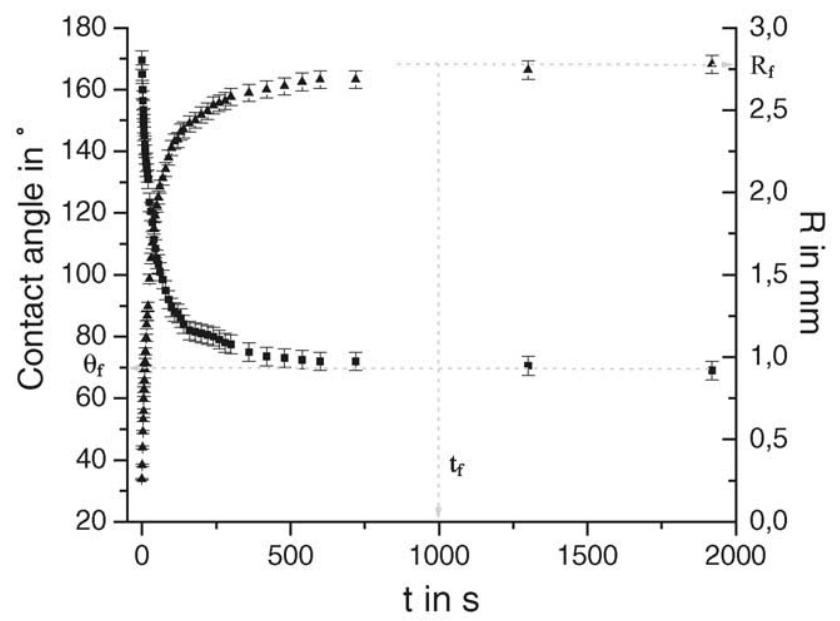

Fig. 2. Contact angle $\left(\theta_{\mathrm{t}}\right)$ and contact area radius $\left(R_{\mathrm{t}}\right)$ as a function of time for wetting of RNOS steel by glass at $T_{\mathrm{w}}=1034^{\circ} \mathrm{C}$.

\subsection{Wetting}

The main feature of the wetting curves in Fig. 2, obtained for $T_{\mathrm{w}}=1034{ }^{\circ} \mathrm{C}$, is the rapid decrease in contact angle during the first $100 \mathrm{~s}$, followed by much slower spreading, leading in about $1000 \mathrm{~s}$ to the final contact angle $\theta_{\mathrm{f}} \approx 70^{\circ}$.

The effect of temperature on wetting curves is illustrated in Fig. 3 for three different values of $T_{\mathrm{w}}$. It may be noted that when $T_{\mathrm{w}}$ increases from 960 to $1200{ }^{\circ} \mathrm{C}$, the final angle is nearly the same: $\theta_{\mathrm{f}}=70 \pm 3^{\circ}$. If $\theta_{\mathrm{f}}$ varies with temperature in this range, it does so within experimental accuracy on $\theta$, i.e. by less than $3^{\circ}$. In contrast, when $T_{\mathrm{w}}$ is increased by $240^{\circ} \mathrm{C}$, the spreading time $\left(t_{\mathrm{f}}\right)$ decreases by one order of magnitude.

The effect of roughness on wetting is shown in Fig. 4, where wetting curves for polished and blasted surfaces can be compared. It appears that the high roughness of the blasted surface (average roughness about of $1.3 \mu \mathrm{m}$ ) has two

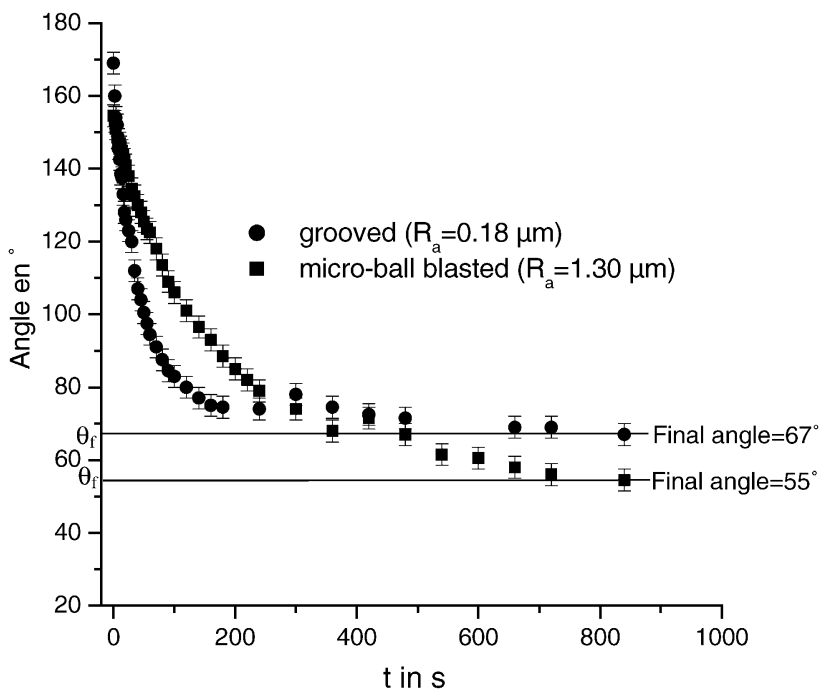

Fig. 4. Contact angle $\left(\theta_{\mathrm{t}}\right)$ as a function of time for grooved and blasted X25V steel surfaces at $T_{\mathrm{w}}=1034^{\circ} \mathrm{C}$.

consequences that are relatively small but reproducible and therefore significant. Firstly, roughness reduces the spreading rate at short times $(t<50 \mathrm{~s})$. Secondly, roughness leads to a decrease in the final contact angle $\left(\theta_{\mathrm{f}}\right)$ by $5-10^{\circ}$. This second effect can be explained by Wenzel's equation [9], which, for systems with $\theta_{\mathrm{f}}<90^{\circ}$ predicts a decrease in the final contact angle with increasing roughness, that is to say with increasing real contact area.

Table 2 summarizes the results concerning the final contact angle of glass on different substrates at $T_{\mathrm{w}}=1034^{\circ} \mathrm{C}$ in a purified helium atmosphere. Contact angles on the three metallic substrates are lower than $90^{\circ}$ and differ only slightly from one another. The wetting observed on the metallic substrates contrasts with the non-wetting found on vitreous carbon, the final contact angle being $135 \pm 5^{\circ}$. It should be noted that the average roughness of $\mathrm{Cv}$ substrates af-

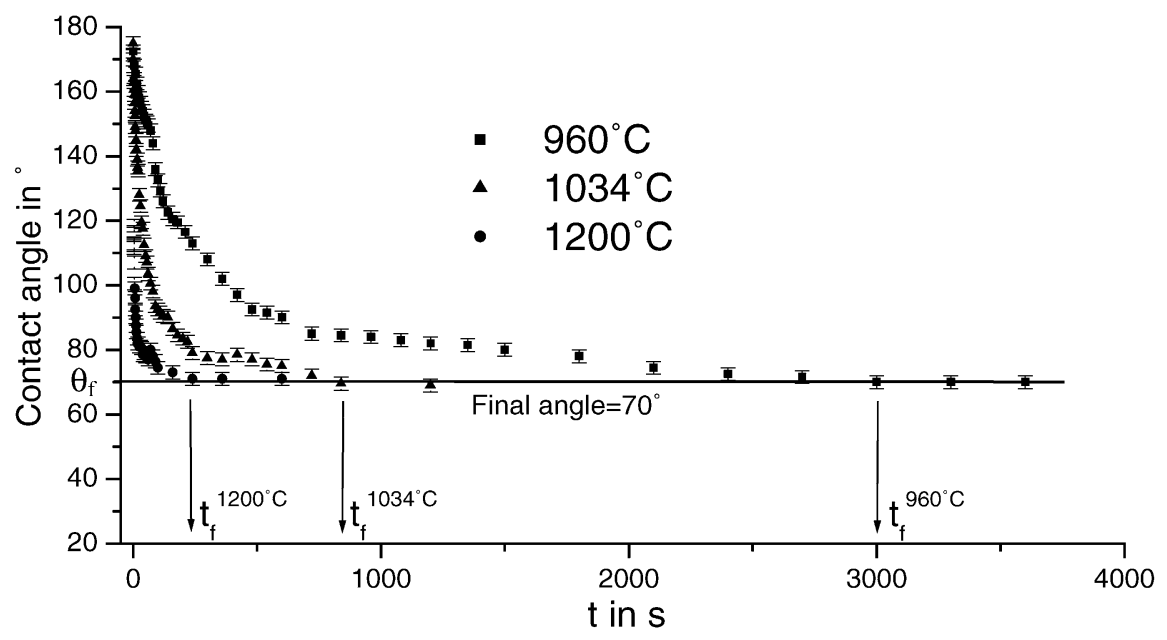

Fig. 3. Contact angle $\left(\theta_{\mathrm{t}}\right)$ as a function of time for wetting of RNOS steel by glass at different temperatures. 
Table 2

Final contact angles $\left(\theta_{\mathrm{f}}\right)$ and detachment temperatures $\left(T_{\mathrm{d}}\right)$ of different glass/substrate systems in purified helium atmosphere for $T_{\mathrm{w}}=1034^{\circ} \mathrm{C}$

\begin{tabular}{lcc}
\hline Material & $\theta_{\mathrm{f}}\left({ }^{\circ}\right)$ & $T_{\mathrm{d}}\left({ }^{\circ} \mathrm{C}\right)$ \\
\hline RNOS steel & $70 \pm 3$ & $170 \pm 20$ \\
X25V steel & $65 \pm 3$ & $100 \pm 20$ \\
$\mathrm{Pt}$ & $75 \pm 3$ & $<20$ \\
$\mathrm{Cv}$ & $135 \pm 5$ & 300 \\
\hline
\end{tabular}

ter the experiments remains at its initial value of a few nanometers.

\subsection{Sticking}

As mentioned above, cooling the glass/substrate system below the vitreous transition temperature $\left(T_{\mathrm{g}} \approx 560{ }^{\circ} \mathrm{C}\right)$ leads in most cases to spontaneous glass separation from the substrate at a well-defined and measurable detachment temperature $\left(T_{\mathrm{d}}\right)$. This separation is driven by relief of stresses generated by the difference in thermal expansion coefficients between the glass and the substrate.

Five experiments were carried out under similar conditions with the RNOS steel substrate, using the same glass mass $(100 \pm 3 \mathrm{mg})$, the same glass refinement temperature $\left(1200^{\circ} \mathrm{C}\right)$ and the same wetting temperature $\left(T_{\mathrm{W}}\right.$ $=1034{ }^{\circ} \mathrm{C}$ ). These experiments all led to a $T_{\mathrm{d}}$ value of 170 $\pm 20^{\circ} \mathrm{C}$. With $T_{\mathrm{w}}=1200$ and $960^{\circ} \mathrm{C}, T_{\mathrm{d}}$ was 171 and $148^{\circ} \mathrm{C}$, respectively; this is within the experimental dispersion of values with $T_{\mathrm{w}}=1034{ }^{\circ} \mathrm{C}$. Therefore, a change in the wetting temperature in the range $960-1200{ }^{\circ} \mathrm{C}$ does not significantly affect the detachment temperature $T_{\mathrm{d}}$.

The $T_{\mathrm{d}}$ values for different glass/substrate systems keeping $T_{\mathrm{w}}$ constant at $1034{ }^{\circ} \mathrm{C}$ are given in Table 2 . The use of $\mathrm{X} 25 \mathrm{~V}$ steel instead of RNOS causes a decrease in $T_{\mathrm{d}}$ from 170 to $100^{\circ} \mathrm{C}$. With platinum, the glass drop remained stuck at room temperature; thus $T_{\mathrm{d}}$ is less than $20^{\circ} \mathrm{C}$ for Pt. Finally the glass $/ \mathrm{Cv}$ system results in the highest detachment temperature, namely $T_{\mathrm{d}}=300^{\circ} \mathrm{C}$.

Experiments carried out using polished (average roughness of $0.18 \mu \mathrm{m}$ ) and blasted (average roughness of $1.3 \mu \mathrm{m}$ ) surfaces of $\mathrm{X} 25 \mathrm{~V}$ steel yielded glass detachment from the substrate at the same temperature of $100^{\circ} \mathrm{C}$.

Intuitively, the energetics of interfacial delamination and hence the detachment temperature $T_{\mathrm{d}}$ must depend on the geometry of the drop/substrate system. For sufficiently wide flat substrates and small (spherical cap shaped) drops, the main pertinent shape parameter is the contact angle $\theta$, which governs in particular the geometric singularity at the triple line. To confirm that this parameter affects $T_{\mathrm{d}}$, two experiments were carried out with the glass/X25V steel system in which glass spreading was interrupted by cooling the sample before capillary equilibrium was attained. These two "interrupted spreading" drops respectively featured $\theta=90^{\circ}, R$ $=2.6 \mathrm{~mm}$ and $\theta=110^{\circ}, R=1.7 \mathrm{~mm}$. For these specimens, $T_{\mathrm{d}}$ was found to be respectively 156 and $158^{\circ} \mathrm{C}$, values that

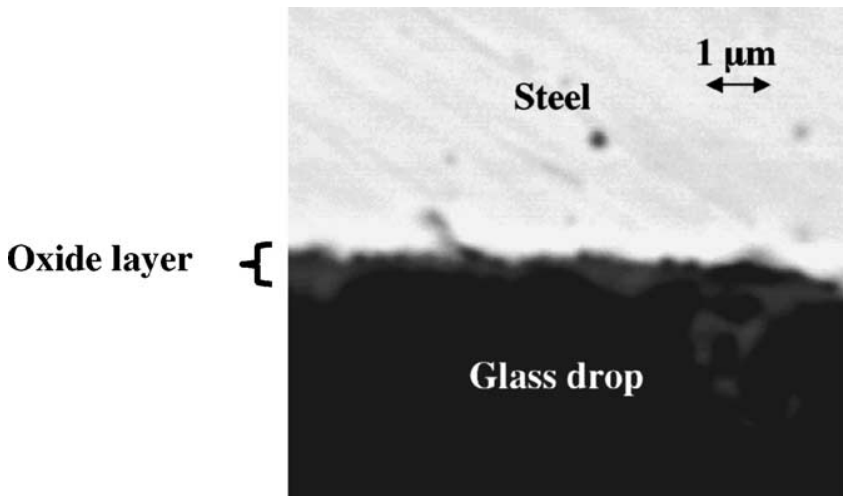

Fig. 5. SEM picture in back-scattered electron mode of glass/oxidized RNOS steel system.

are higher than the $T_{\mathrm{d}}$ value of $100 \pm 20^{\circ} \mathrm{C}$ obtained with a glass drop on $\mathrm{X} 25 \mathrm{~V}$ having the final equilibrium contact angle $\theta_{\mathrm{f}}$ of $65 \pm 2^{\circ}$. The $T_{\mathrm{d}}$ value is thus indeed sensitive to the drop geometry.

An oxide film, 1-2 nm thick, naturally covers stainless steel surfaces in air at room temperature. During heating to the wetting temperature in the neutral atmosphere used here, this film was wholly removed by deoxidization, so that glass wetting was measured on non-oxidized steel surfaces. To study the effect of surface oxidation on sticking, a RNOS steel surface covered by an oxide layer $1 \mu \mathrm{m}$ thick (see Section 2) was used in a separate experiment. To minimize oxide volatilization and dissolution into the glass during the experiment, the wetting temperature was lowered to $860^{\circ} \mathrm{C}$. In view of the low rate of drop spreading at this temperature, the experiment was stopped before capillary equilibrium could be reached, at an instantaneous $\theta$ value of $105^{\circ}$. As with a platinum substrate, this glass drop adhered to the substrate throughout the cooling cycle, down to room temperature $\left(T_{\mathrm{d}}<20^{\circ} \mathrm{C}\right)$. During subsequent storage, this sample showed spontaneous internal cracking of the glass drop along a cone aligned with the drop axis. A continuous layer of oxide was still present along the glass/steel interface of this sample after the experiment, Fig. 5.

\subsection{Interface characterization}

The glass/steel specimens exhibited two different behaviors during cooling, as mentioned above. With the oxidized steel, sticking down to room temperature was observed. With non-oxidized steel, detachment was observed before reaching room temperature. Fig. 6 presents the free surface of the RNOS steel around the zone of contact with the glass. The grain boundaries are more accentuated inside the zone of contact. A comparison of the roughness profiles of each zone, presented in Fig. 7, shows that this accentuation is due to the formation of grain boundary grooves. These are deeper inside the zone of contact, indicating that contact with glass promotes steel grain boundary grooving. This observation is confirmed by AFM measurements of groove geometry: 


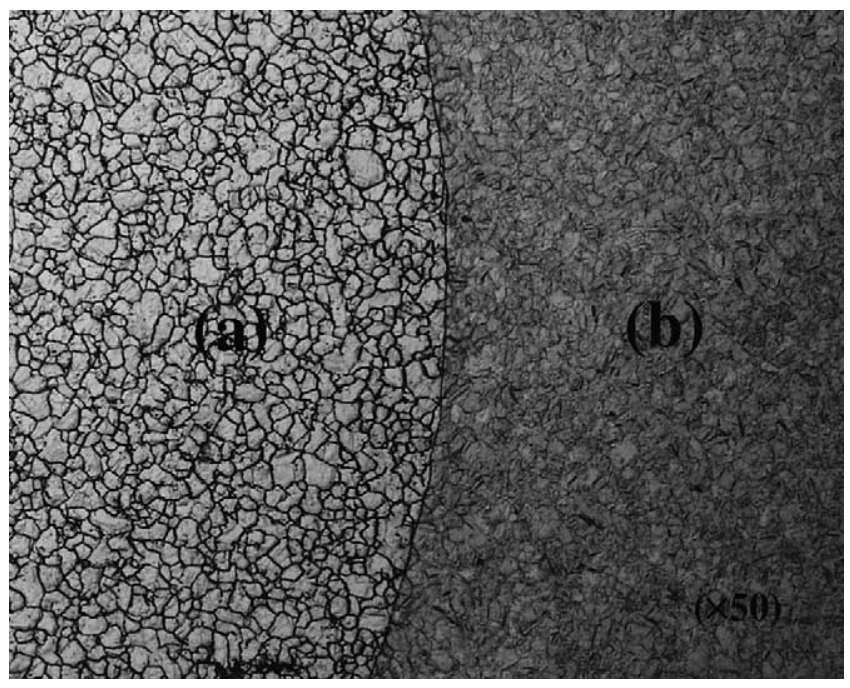

Fig. 6. Optical micrograph of grooved RNOS steel surface after glass drop detachment for $T_{\mathrm{w}}=1034^{\circ} \mathrm{C}$ : (a) surface formerly in contact with glass; (b) free surface. along the free surface the grooves are $0.15 \pm 0.05 \mu \mathrm{m}$ deep and $1.6 \pm 0.4 \mu \mathrm{m}$ wide, whereas in the zone of contact the grooves are $0.8 \pm 0.1 \mu \mathrm{m}$ deep and $6.1 \pm 0.5 \mu \mathrm{m}$ wide, respectively. On the basis of AFM analysis of the glass and steel contact surfaces, it clearly appears that the molten glass espouses perfectly the shape of grooves (see Fig. 8a). Moreover the average roughness $\left(R_{\mathrm{a}}\right)$ of the glass and steel areas between the grooves is very close, at about $10 \mathrm{~nm}$ (see Fig. 8b). For comparison, $R_{\mathrm{a}}$ of a glass surface that has not been in contact with steel is only $1-3 \mathrm{~nm}$. In addition, molten glass totally penetrates hollows a few micrometers in depth formed by micro-ball blasting, as seen on Fig. $9 \mathrm{~b}$.

On a macroscopic scale, glass/steel separation occurs along the interface. The glass was found not to contain constituents of the steel along the surface of former contact. When the interior of the metal grains was analyzed by SEM-EDX spectrometry, no constituents of the glass were found either; however, some glass constituents $(\mathrm{Si}, \mathrm{Na}$ and $\mathrm{Ca}$ ) were detected in the grain boundary grooves, as seen in Fig. 10. AFM characterization shows that the presence of

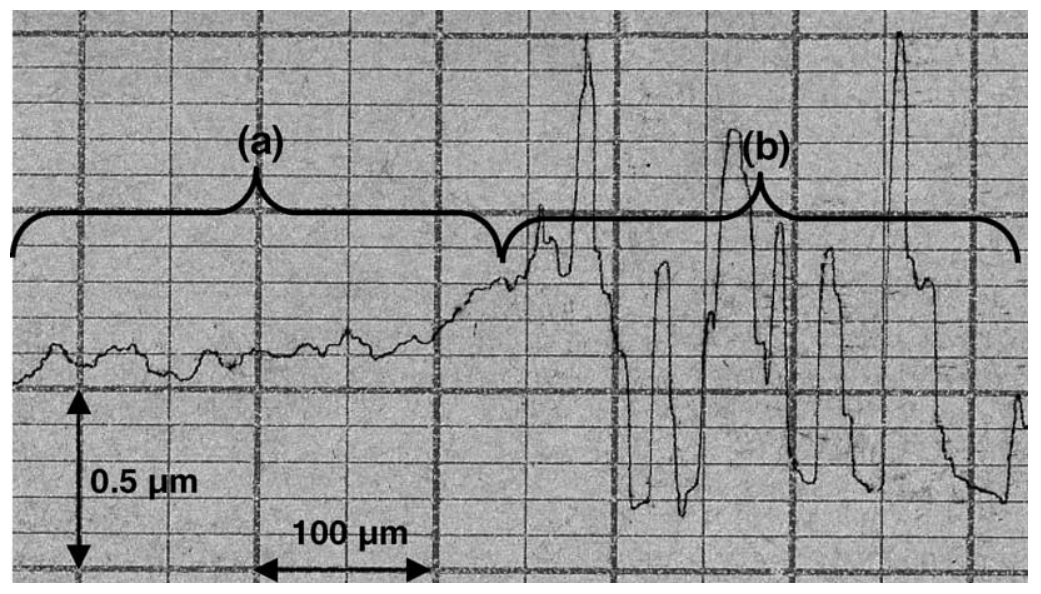

Fig. 7. Roughness profiles of grooved RNOS steel surface for $T_{\mathrm{w}}=1034^{\circ} \mathrm{C}$ : (a) free surface; (b) surface formerly in contact with glass.

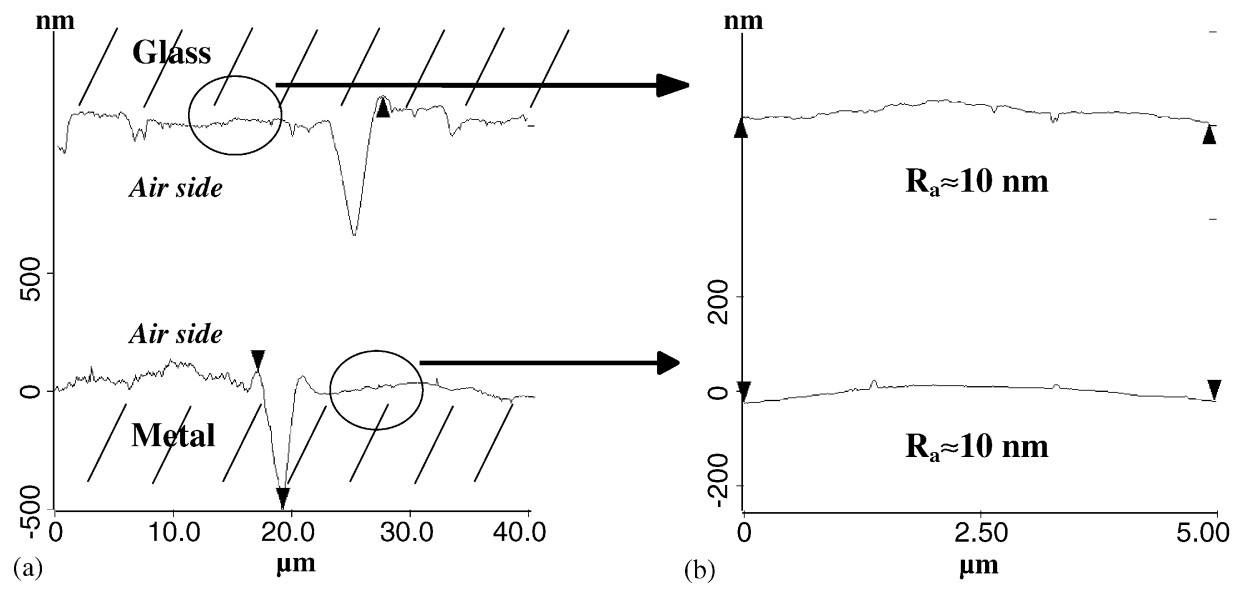

Fig. 8. (a) AFM profiles of grain boundary grooves on glass and RNOS steel surfaces and (b) local average roughness for $T_{\mathrm{w}}=1034^{\circ} \mathrm{C}$. 

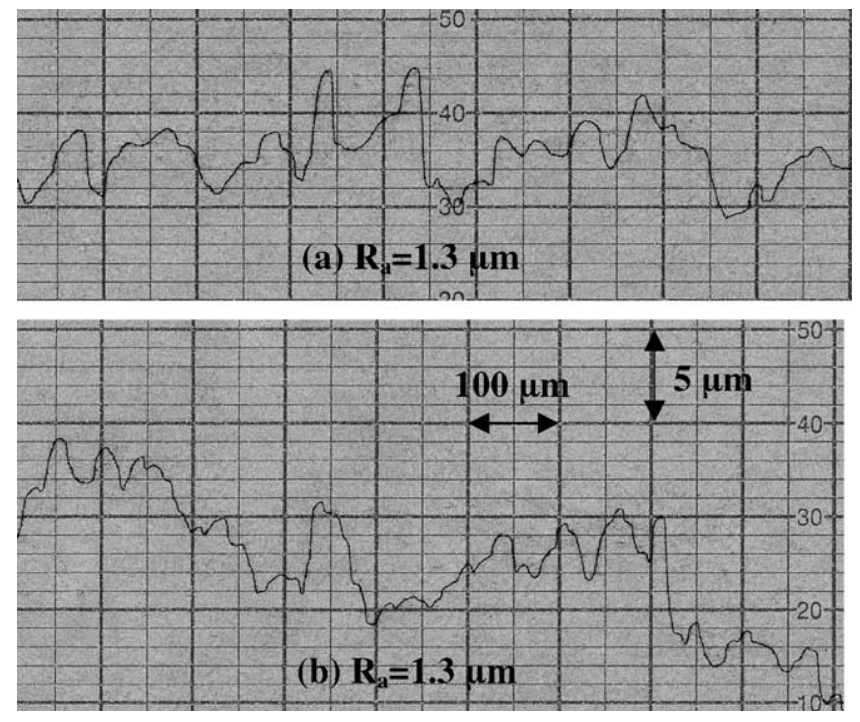

Fig. 9. Talysurf surface profiles of (a) X25V steel and (b) glass surfaces after micro-ball blasting for $T_{\mathrm{w}}=1034{ }^{\circ} \mathrm{C}$.

these constituents is due to glass particles that are retained in the grooves. Therefore, even if the overall rupture is of the adhesive type, it seems to be partly cohesive in the vicinity of the grain boundary grooves.

From the above results it is concluded that no reaction is detectable by SEM-EDX spectrometry in glass/non-oxidized steel systems. This conclusion is only valid for $T \geq 960^{\circ} \mathrm{C}$, since oxidation/reduction reactions occur between the glass and the steel at $T \leq 860^{\circ} \mathrm{C}$, as seen at the beginning of Section 3. But even at $T \geq 960^{\circ} \mathrm{C}$ some limited glass/steel interactions must exist, as testified by a change from uncolored soda-lime glass to blue (RNOS) or violet $(\mathrm{X} 25 \mathrm{~V})$ glass. It should be noted that similar changes in glass color are obtained by adding metallic oxides at ppm levels ([10], p. 196) and that the blue coloration can be attributed to the formation and the dissolution of $\mathrm{CrO}$ into the glass [11].

\section{Discussion}

\subsection{Wetting}

Non-reactive wetting of oxides by molten metals is observed for couples in which mass transfer through the interface is very limited and has a negligible effect on the interfacial energies. Criteria for identifying a metal/oxide system as non-reactive are discussed in [12]. A strong indication of non-reactive wetting is given by a very small variation in final contact angle with temperature, typically $-0.01^{\circ} \mathrm{K}^{-1}$. The same behavior was found here for the molten soda-lime glass/RNOS steel system: the final contact angle $\left(\theta_{\mathrm{f}}\right)$ hardly varies with temperature within the range 960-1200 ${ }^{\circ} \mathrm{C}$. This result, together with the interface characteristics, leads to the conclusion that molten soda-lime glass/stainless steel systems are non-reactive from the point of view of wetting above $960{ }^{\circ} \mathrm{C}$ in a neutral atmosphere. If this conclusion is true for metallic substrates containing alloying elements having a high affinity for oxygen such as chromium, it is a fortiori true for a noble metal such as Pt.

Molten soda-lime glass does not wet a $\mathrm{Cv}$ substrate, the measured final contact angle $\left(\theta_{\mathrm{f}}\right)$ being in the $130-140^{\circ}$ range. With a surface energy of molten soda-lime glass, $\sigma_{\mathrm{LV}}$ $\approx 0.35 \mathrm{~J} \mathrm{~m}^{-2}$ ([10], p. 271), the calculated work of adhesion, $W_{\mathrm{a}}=\sigma_{\mathrm{LV}}\left(1+\cos \theta_{\mathrm{f}}\right)$, is about $0.1 \mathrm{~J} \mathrm{~m}^{-2}$. These $\theta_{\mathrm{f}}$ and $W_{\mathrm{a}}$ values are typical of systems in which adhesion is ensured by van der Waals' interaction alone ([13], p.320).

Molten soda-lime glass wets metallic substrates well. With $\theta_{\mathrm{f}}=70^{\circ}, W_{\mathrm{a}}$ is about $0.5 \mathrm{~J} \mathrm{~m}^{-2}$. Both the $\theta_{\mathrm{f}}$ and $W_{\mathrm{a}}$ values are very different from those obtained for molten soda-lime glass on vitreous carbon. The first hypothesis is to suppose that, even in non-reactive glass/metal systems, adhesion may be due to chemical interactions that are entirely localized at the common interface. In the case of steel substrates, such interactions could take place between the oxygen of the glass and the chromium of the steel, a metallic element that has a strong affinity with oxygen; however, similar $\theta_{\mathrm{f}}$ and $W_{\mathrm{a}}$ values were obtained for molten soda-lime glass on Pt, a metal that is not expected to develop chemical interactions with an oxide in a neutral atmosphere.

In sessile drop experiments performed by Pask and co-workers with a molten $66 \% \mathrm{SiO}_{2}-34 \% \mathrm{Na}_{2} \mathrm{O}$ sodium silicate glass (which is similar to soda-lime glass) on various metals $(\mathrm{Cu}, \mathrm{Ag}, \mathrm{Au}$ and $\mathrm{Pt})$ under helium, the final contact angles were found to be in the $60-70^{\circ}$ range [14]. This agrees with the present results, showing that the wetting of metals by molten glass in neutral gas atmospheres appears not to be very sensitive to the nature of the metallic substrate. This strongly suggests that adhesion in non-reactive glass/metal systems is ensured mainly by physical interactions.

Exactly the same conclusion has been drawn from analyses of wetting data for non-reactive systems consisting of a liquid metal drop, such as $\mathrm{Cu}, \mathrm{Ag}$, $\mathrm{Au}, \mathrm{Ni}$ and $\mathrm{Fe}$, on ionocovalent oxides such as $\mathrm{Al}_{2} \mathrm{O}_{3}, \mathrm{SiO}_{2}$ and $\mathrm{MgO}$ ([13], p. 207). In these systems, final contact angles are in the $120-130^{\circ}$ range. Taking a surface energy of $1.7 \mathrm{~J} \mathrm{~m}^{-2}$ for a Fe-rich alloy ([13], p.148) this leads to a work of adhesion $\left(W_{\mathrm{a}}\right)$ of $0.7 \mathrm{~J} \mathrm{~m}^{-2}$, which is close to the value of $0.5 \mathrm{~J} \mathrm{~m}^{-2}$ found for molten soda-lime glass on steels. These similar values of $W_{\mathrm{a}}$ imply that the different wetting results of molten Fe-rich alloys on oxide substrates $\left(\theta_{\mathrm{f}} \approx 120-130^{\circ}\right)$ and of molten soda-lime glass on steels $\left(\theta_{\mathrm{f}} \approx 70^{\circ}\right)$ are only due to the different $\sigma_{\mathrm{LV}}$ values: about 1.7 and $0.35 \mathrm{~J} \mathrm{~m}^{-2}$ for molten steel and molten glass, respectively.

The above considerations are only valid for glass on non-oxidized metallic surfaces. An enhancement in wetting can occur if the metal surface has been oxidized, as this leads to the development of chemical bonding across the interface. Perfect or nearly perfect wetting is then observed ([13], p.341). 


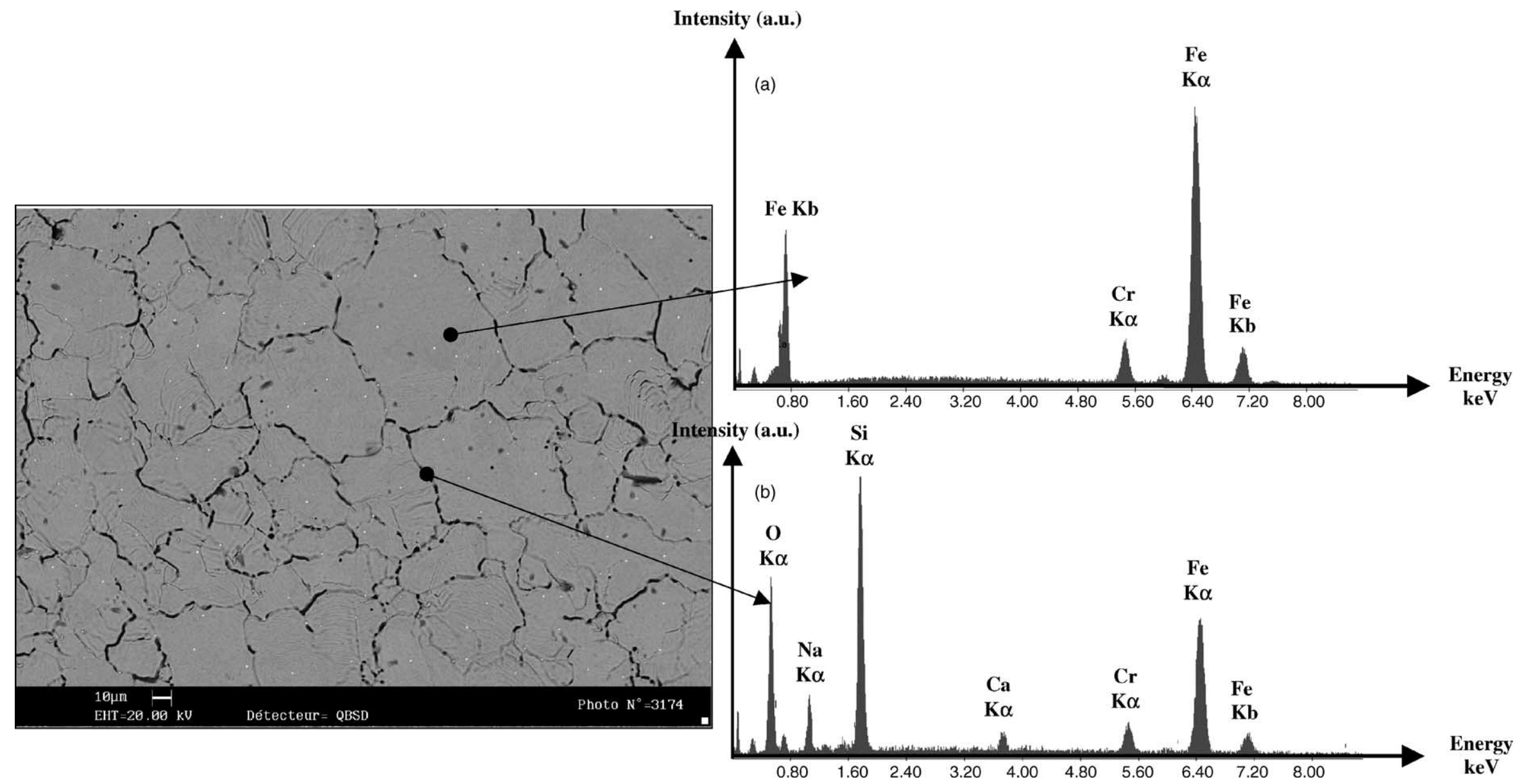

Fig. 10. SEM picture in back-scattered electron mode of grooved RNOS steel surface formerly in contact with glass; (a) EDX analysis of a grain interior (metal elements identified: Fe, Cr and Ni); (b) EDX analysis of a grain boundary groove (glass and metal elements identified: $\mathrm{Si}, \mathrm{Na}, \mathrm{Ca}, \mathrm{Fe}$ and $\mathrm{Cr}$ ). 
For non-reactive liquid metals on ceramic or metallic substrates, the time needed for millimeter-sized drops to reach the final contact angle is very short, on the order of $0.01 \mathrm{~s}$ [15]. As Fig. 3 shows, glass spreading can continue for minutes and even tens of minutes depending on the temperature. Obviously this is because the viscosity of glasses $(\eta)$ is several orders of magnitude higher than that of metals. For instance $\eta$ of soda-lime glass is about $360 \mathrm{~Pa}$ s at $1100{ }^{\circ} \mathrm{C}$ [16], whereas molten $\mathrm{Au}$ and $\mathrm{Cu}$ have a viscosity of a few $\mathrm{mPas}$ at the same temperature. Extensive experimental studies have been performed at room temperature with low-temperature liquids, such as polymers or silicon oils. These investigations showed that, in the case of liquids producing perfect wetting, the experimental values of the instantaneous contact angle $\theta$ plotted as a function of the reduced spreading rate, $z=\left(\eta / \sigma_{\mathrm{LV}}\right)(\mathrm{d} R / \mathrm{d} t)$, lie on a single curve [17]. The final contact angle for molten soda-lime glass/steel systems between 960 and $1200^{\circ} \mathrm{C}$ is not zero, but it is nearly constant at about $70^{\circ}$. In Fig. 11, we can see that, here too, the experimental points lie on the same $\theta(z)$ curve whatever the temperature. Since the coefficient of variation of $\sigma_{\mathrm{LV}}$ with temperature is very weak, typically as low as $10^{-4} \mathrm{~J} \mathrm{~m}^{-2} \mathrm{~K}^{-1}$ [17], the curve in Fig. 11 suggests that the glass spreading rate is governed by viscous friction. A more detailed discussion of spreading kinetics in glass/metal systems will be presented elsewhere [18].

\subsection{Sticking}

As detailed above, there is a marked difference across material pairs investigated here in the temperature $T_{\mathrm{d}}$ at which the solidified drop detaches spontaneously from the substrate. It is tempting to use this observation and the as- sociated data for an evaluation of sticking from system to system. To this end, we adopt a crude approach, based on simplified energetic arguments.

A linear elastic bimaterial edge such as the region surrounding a sharp triple line is known to be a site of stress singularity (e.g. [19,20] and references therein). For the geometry and materials of present interest, this is confirmed by finite-element simulations conducted by one of the present authors (M. Braccini, work in progress). Also, by analogy with the case of a thin film with $\theta=\pi / 2$, which has been analyzed extensively given its practical importance, initial propagation of a very small crack nucleated along the triple line is expected to be driven by roughly the same rate of elastic energy release $(G)$ as a long interfacial crack [21,22]. These facts taken together indicate that crack nucleation is not expected to be limiting. We therefore attempt to differenciate between samples by focusing on the energetics of crack propagation during drop interfacial detachment; to this end, we take a very simplified approach.

Clearly, the driving force for drop detachment is in the elastic energy that builds up during cooling due to thermal contraction mismatch between the substrate and the drop. A simple expression is proposed in [23] for the stored elastic energy $U_{\mathrm{e}, \mathrm{A}}$ built-up due to differential contraction in an elastic cylinder of material (A), radius $r$ and height $h$ that is bonded end-on-end to another elastic cylinder of the same radius but made of a different material (B), after a change $\Delta T$ in temperature from an initial stress-free state:

$$
U_{\mathrm{e}, \mathrm{A}}=\frac{E_{\mathrm{A}} E_{\mathrm{B}}^{2}}{\left(E_{\mathrm{A}}+E_{\mathrm{B}}\right)^{2}}\left(\alpha_{\mathrm{A}}-\alpha_{\mathrm{B}}\right)^{2} \Delta T^{2} r^{3} \operatorname{erf}\left(\frac{h}{r}\right),
$$

where subscripts $\mathrm{A}$ and $\mathrm{B}$ denote properties of materials $\mathrm{A}$ and $\mathrm{B}$ respectively, $\alpha$ the coefficient of thermal expansion,

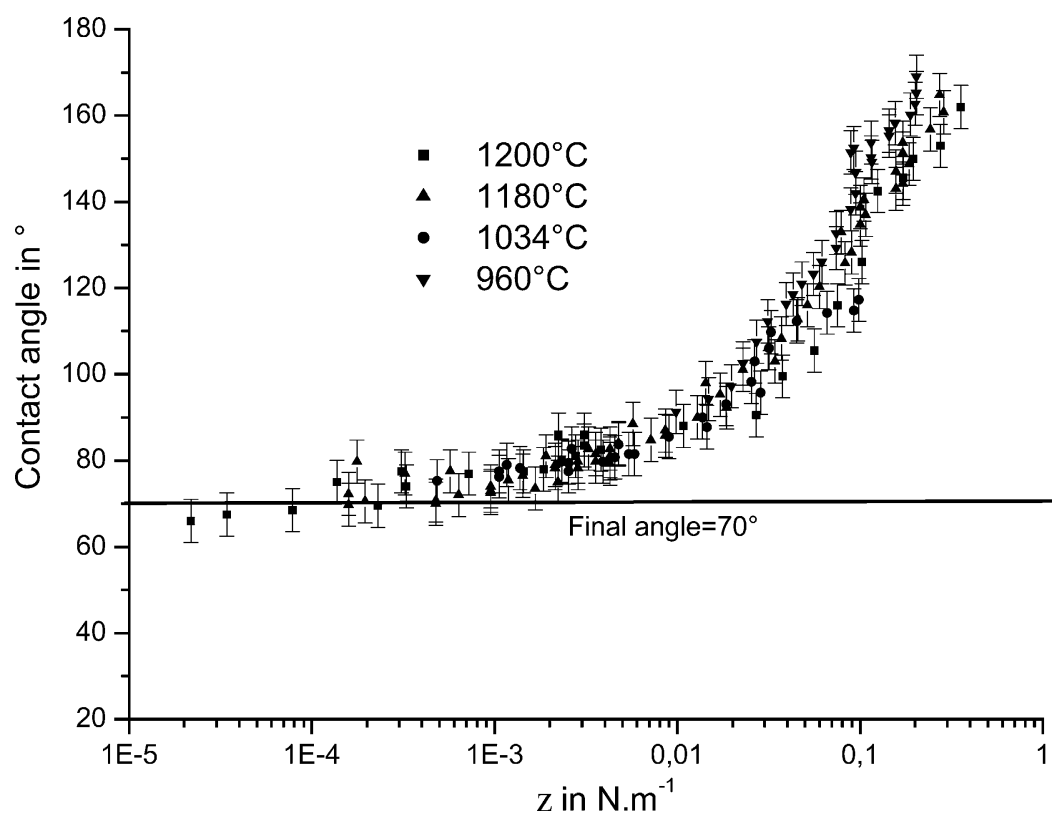

Fig. 11. Contact angle $(\theta)$ as a function of the reduced spreading rate $z=\eta U / \sigma_{\mathrm{LV}}$ for RNOS steel wetting by glass at different temperatures. 


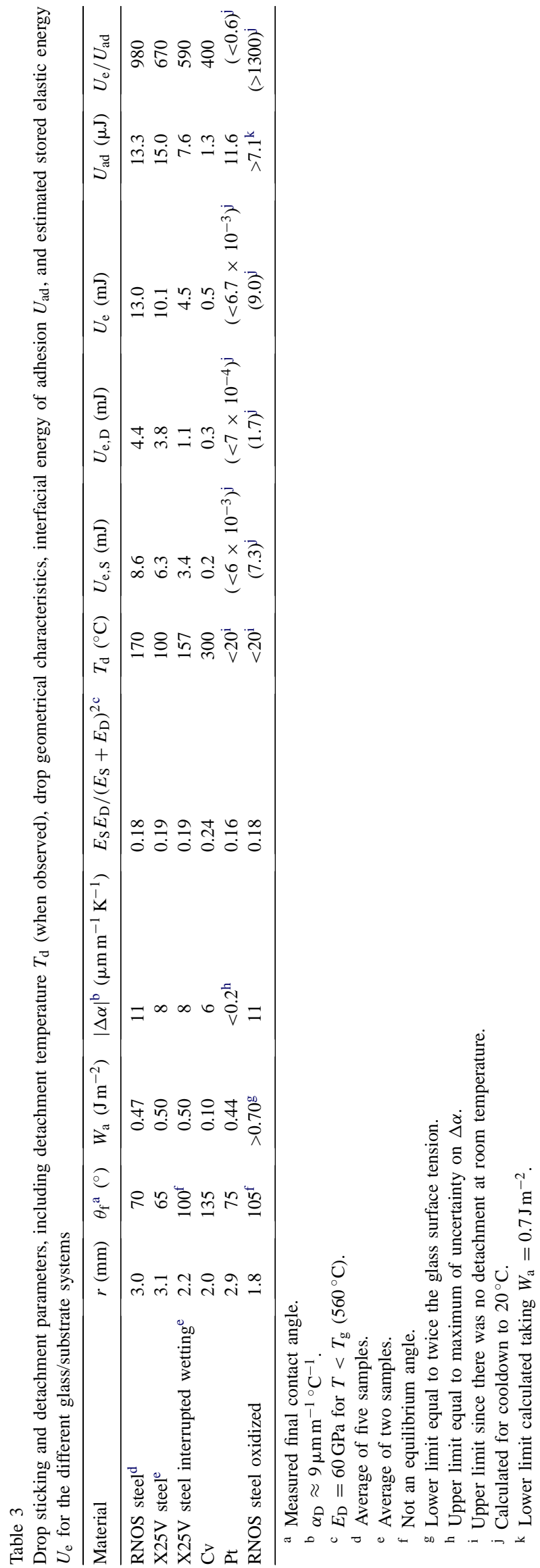

$E$ Young's modulus, and $\operatorname{erf}(x)$ the error function. In the present experiments, at the moment of drop detachment, $\Delta T$ $=T_{\mathrm{g}}-T_{\mathrm{d}}$ where $T_{\mathrm{g}} \approx 560^{\circ} \mathrm{C}$. We assimilate the glass drop to a cylinder having the same circular cross-section $\pi r^{2}$ as the drop/substrate interfacial contact area and of height $h$ giving the appropriate drop volume, and the substrate to a cylinder of equal radius and height $h$ equal to the substrate thickness. The total stored elastic energy $U_{\mathrm{e}}=U_{\mathrm{e}, \mathrm{S}}+U_{\mathrm{e}, \mathrm{D}}$ (where subscripts $\mathrm{S}$ and D stand for substrate and drop, respectively) at the moment of drop detachment can then be estimated using the above expression; values are given in Table 3. Steel substrate yield stresses (above $400 \mathrm{MPa}$ at temperatures up to $600^{\circ} \mathrm{C}$ ) are sufficiently high that the estimated stored energy is changed by only $50 \%$ if substrate yielding is taken into account using results of [23]; for simplicity we ignore this effect.

Table 3 shows that the ratio of the estimated stored elastic energy $U_{\mathrm{e}}$ at the moment of drop detachment to the measured interfacial energy of adhesion $U_{\text {ad }}$ :

$U_{\mathrm{ad}}=\pi r^{2} W_{\mathrm{a}}=\pi r^{2} \sigma_{\mathrm{LV}}\left(1+\cos \theta_{\mathrm{f}}\right)$

is relatively constant, varying between 400 and 980 , Table 3 (for comparison, $U_{\mathrm{ad}}$ varies by one order of magnitude). This can be explained if we assume that (i) the driving force for crack propagation (namely the rate of release of elastic energy $G$ ) upon drop detachment is proportional to the stored elastic energy $U_{\mathrm{e}}$ per unit interface area and (ii) that the critical value of $G$ is proportional to the work of adhesion $W_{\mathrm{a}}$. We also note that, if most of the stored elastic energy is consumed in crack propagation, observed ratios of $U_{\mathrm{e}} / U_{\mathrm{ad}}$ should be of the same order of magnitude as the ratio $G / W_{\mathrm{a}}$. Observed values of $U_{\mathrm{e}} / U_{\mathrm{ad}}$ are, indeed, of the same order of magnitude (between $10^{2}$ and $10^{3}$ ) as measured ratios of metal/ceramic interfacial fracture energy to interfacial work of adhesion [24] or of the fracture energy of cleaving metals to their surface energy [25]. We thus use this simplified approach to interpret sample-to-sample variations in the drop detachment temperature $T_{\mathrm{d}}$.

With non-oxidized steels, $T_{\mathrm{d}}$ values are within the range $100-170^{\circ} \mathrm{C}$. Variations are not far from experimental error (of $40^{\circ} \mathrm{C}$ ); they are also too small to be reasonably accounted for by as simple a calculation as that presented here. We note nonetheless that the slight increase in detachment temperature that is found with the interrupted (higher apparent $\theta$ ) experiments on $\mathrm{X} 25 \mathrm{~V}$ steel is roughly explained as the result of a smaller contact area for a constant drop volume.

With a Pt substrate, sticking is preserved at room temperature: this is explained by the very low value of $\Delta \alpha$ for the glass/Pt couple, Table 3 . With vitreous carbon substrates, detachment is observed after a temperature excursion of $260^{\circ} \mathrm{C}$ despite the very low work of adhesion: this is due to the low elastic modulus of the substrate, causing the rate of increase of stored elastic energy with increasing $\Delta T$ to be far lower than with steel substrates.

With a RNOS substrate, the detachment temperature is lowered from $170^{\circ} \mathrm{C}$ for non-oxidized steel to less than 
$20^{\circ} \mathrm{C}$ for oxidized steel. This can be attributed, at least in part, to the increase in $W_{\mathrm{a}}$ that accompanies the change in glass/metal interface nature from physical to chemical bonding. For a work of adhesion $W_{\mathrm{a}}$ of $0.7 \mathrm{~J} \mathrm{~m}^{-2}$, equal to twice the glass surface energy $\sigma_{\mathrm{LV}} \approx 0.35 \mathrm{~J} \mathrm{~m}^{-2}$ ([10], p. 271), the detachment temperature can be estimated by the present calculation assuming a ratio of the stored elastic energy to the interfacial energy of adhesion $\left(U_{\mathrm{e}} / U_{\mathrm{ad}}\right)$ equal to $10^{3}$ consistent with previous results. The predicted $T_{\mathrm{d}}$ is then about $100^{\circ} \mathrm{C}$ and not $20^{\circ} \mathrm{C}$ or below for oxidized RNOS steel. This explains the room-temperature sub-critical cracking of the glass that was observed in these samples, but also indicates that additional factors must intervene to prevent drop detachment during cool-down, as this could occur by fracture through the glass along the interface. Such factors may include modification of the glass near the interface by chemical interaction with the oxide (see above, Section 3.3), the mechanical influence of the intermediate layer of oxide between the glass and the substrate, or environmental effects.

Overall, it is significant that, whatever the steel surface state, be it oxidized or non-oxidized, $\Delta T=T_{\mathrm{g}}-T_{\mathrm{d}}$ is high, reaching several hundred degrees. In glass container production, low values are needed: the centrifugation process requires for instance $\Delta T$ values of only a few $\mathrm{K}$ or tens of $\mathrm{K}$, depending on process specifics. These far lower values of $\Delta T$ obtained in industrial practice are due to differences in the microscopic configuration at the glass/metallic mold interface. Partial interfacial contact obtains in industrial specimens, in contrast to the true interfaces of full metal/glass contact that are generated with the present sessile drop experiments. This partial interfacial contact is, in turn, a combined result of the high cooling rates applied in industrial processing and the high viscosity of molten glass. The question is examined in detail elsewhere [26].

\section{Conclusion}

Characterization of stainless steel and glass surfaces after separation, using a surface profilometer, AFM and SEM with an EDX spectrometer, leads to the conclusion that there is no significant chemical reaction between glass and stainless steel in a neutral gas environment and at $T \geq 960^{\circ} \mathrm{C}$. The main effect of glass/steel interaction is an enhancement of grain boundary grooving on the stainless steel surface.

Wetting experiments involving molten soda-lime glass on different substrates, performed by the "transferred drop" version of the sessile drop technique, show that the glass wets stainless steel with a final contact angle $\left(\theta_{\mathrm{f}}\right)$ close to $70^{\circ}$. Temperature has a negligible effect on $\theta_{\mathrm{f}}$ but strongly affects the spreading rate. As with non-reactive liquid metal/ionocovalent oxide systems, interfacial bonding governing wetting and adhesion in these glass/metal systems appears to involve only physical force contributions.

Whatever the temperature in the range $960-1200^{\circ} \mathrm{C}$, all instantaneous contact angles $(\theta)$ versus reduced spreading rate $z=\left(\eta / \sigma_{\mathrm{LV}}\right)(\mathrm{d} R / \mathrm{d} t)$ obtained for the same glass/metal couple lie on a single curve. This suggests that viscous friction alone governs spreading kinetics.

Sticking is quantified by the difference in temperature $\Delta T$ between the vitreous transition temperature and the temperature of glass detachment from the substrate. For non-oxidized stainless steels, $\Delta T$ is several hundred $\mathrm{K}$. An enhancement of sticking is observed with Pt and with oxidized stainless steels, leading in both cases to sticking down to room-temperature. A simple energy balance is used to relate $\Delta T$ to the difference in thermal expansion coefficient between the substrate and the glass $(\Delta \alpha)$, to the elastic parameters and geometry of contacting phases, as well as to the work of adhesion $\left(W_{\mathrm{a}}\right)$ of the glass/substrate system.

\section{Acknowledgements}

This research was supported by the French Ministry of Economy, Finances and Industry under the program no. 004906079. We wish to thank Eng. L. Bedel, L. Federzoni and E. Rigal of CEA Grenoble (France) for their helpful contributions in interpreting sticking.

\section{References}

[1] J. Zarzycki, Les verres et l'état vitreux, Masson, Paris, 1982, p. 359.

[2] A.J.H.P. Van der Pol, T.R. Mulderij, in: GLAFO (Ed.), Proceedings of the Conference on Fundamentals of Glass Science and Technology, Vaexjoe, Sweden, 9-12 June 1997, Swedish Glass Research Institute, Växjö, Sweden, p. 389.

[3] D. Zhong, E. Mateeva, I. Dahan, J.J. Moore, G.G.W. Mustoe, T. Ohno, J. Disam, S. Thiel, Surf. Coat. Techn. 133/134 (2000) 8.

[4] W. Trier, F. Hassoun, Glastech. Ber. 45 (1972) 271.

[5] H.V. Fairbanks, in: Proceedings of the Symposium on Hot Glass Contact with Metal, USCV, Charleroi, Belgium, 1984, p. 575.

[6] P. Manns, W. Döll, G. Kleer, Glastech. Ber. Glass Sci. Technol. 68 (1995) 389.

[7] M. Falipou, C. Donnet, F. Marechal, J. Charenton, Glastech. Ber. Glass Sci. Technol. 70 (1997) 137.

[8] Smithells Metals Ref. Book, 6th ed., Brandes, 1983, pp. 14-22.

[9] R.N. Wenzel, Ind. Eng. Chem. 28 (1936) 988.

[10] H. Scholze, Le verre: nature, structure et propriétés, 2nd ed., Institut du verre, Paris, 1980.

[11] A.P. Tomsia, Z. Feipeng, J.A. Pask, J. Am. Ceram. Soc. 68 (1985) 20.

[12] N. Eustathopoulos, B. Drevet, Mater. Sci. Eng. A 249 (1998) 176.

[13] N. Eustathopoulos, M. Nicholas, B. Drevet, in: R.W. Cahn (Ed.), Wettability at High Temperatures, Pergamon Materials Series, vol. 3, Pergamon, Oxford, 1999.

[14] V.F. Zackay, D.W. Mitchell, S.P. Mitoff, J.A. Pask, J. Am. Ceram. Soc. 36 (1953) 84.

[15] P. Protsenko, A. Terlain, M. Jeymond, N. Eustathopoulos, J. Nucl. Mater. 307 (2002) 1396.

[16] T. Lakatos, L.G. Johansson, B. Simmingsköld, Glass Technol. 13 (1972) 88.

[17] S.F. Kistler, in: J.C. Berg (Ed.), Wettability, Marcel Dekker, New York, 1993, p. 311.

[18] J. Pech, in preparation.

[19] J.P. Dempsey, G.B. Sinclair, J. Elasticity 11 (1981) 317. 
[20] X.H. Liu, Z. Suo, Q. Ma, Acta Mater. 47 (1999) 67.

[21] H.H. Yu, M.Y. He, J.W. Hutchinson, Acta Mater. 49 (2001) 93.

[22] L.B. Freund, S.S. Suresh, Thin Film Materials, Stress, Defect Formation and Surface Evolution, Cambridge University Press, Cambridge, UK, 2003, p. 220.

[23] J.W. Park, P.F. Mendez, T.W. Eagar, Acta Mater. 50 (2002) 883.
[24] A.G. Evans, J.W. Hutchinson, Y. Wei, Acta Mater. 47 (1999) 4093.

[25] M.F. Ashby, D.R.H. Jones, Engineering Materials-An Introduction to their Properties and Applications, vol. 1, 2nd ed., Butterworths/Heinemann, Oxford, 1996, p.136, Fig. 13.5.

[26] J. Pech, submitted. 